\title{
Etude de la dispersion de vitesse d'une onde ultrasonore dans un composite stratifié
}

\author{
C. Gazanhes, J. P. Hérault, M. Miane, H. Saul \\ Equipe Ultrasons, Laboratoire de Mécanique et d'Acoustique du C.N.K.S., \\ 31, chemin Joseph Aiguier, B.P. 71, 13277 Marseille Cedex 9, France
}

et A. Ben Chaala

Centre des Sciences et de la Technologie Nucléaire, O.N.R.S., Bd. Frantz Fanon, B.P. 1017, Alger Gare, Algérie

(Reçu le 21 mai 1982, révisé le 4 octobre 1982, accepté le 14 octobre 1982)

\begin{abstract}
Résumé. - On étudie la dispersion de vitesse d'une onde ultrasonore se propageant parallèlement aux couches d'un milieu stratifié à deux composants (aluminium-résine époxyde) dans la gamme de fréquences $0,1-2,5 \mathrm{MHz}$. On a mis en évidence deux modes de propagation : un mode lent ne possédant pas de fréquence de coupure, et un mode rapide possédant une fréquence de coupure qui dépend de l'épaisseur et de la nature des couches. On compare les résultats expérimentaux à trois modèles théoriques dont on évalue les domaines de validité.
\end{abstract}

\begin{abstract}
The dispersion of ultrasonic waves by laminated composites is studied by investigating the propagation parallel to the layers of a two constituents aluminium-epoxy composite material, in the frequency range 0.1-2.5 MHz. Two modes of propagation, with different propagation velocity, were observed. The slow velocity propagation mode has a finite propagation velocity for $\omega \rightarrow 0$. The high velocity propagation mode has an infinite propagation velocity for some cut-off frequency, value of which is determinated by the thickness and the nature of the layers. The comparison and discussion of the experimental data with the prediction from three theoretical models are presented.
\end{abstract}

1. Introduction. - Depuis une vingtaine d'années, les technologies modernes de l'espace, de l'aéronautique, de l'électronique, de la biomécanique... etc., font appel aux matériaux composites.

- Les composites sont constitués par une matrice englobant un matériau de renforcement. La matrice donne sa cohésion au composite et transmet les charges au matériau de renforcement. Le but des composites est donc de tirer parti des propriétés mécaniques des matériaux assemblés par la matrice afin d'obtenir un nouveau matériau ayant des propriétés mécaniques performantes que ne possèdent pas les composants principaux.

- Les caractéristiques mécaniques du composite dépendent de la nature des composants, mais aussi de la géométrie et de la disposition des matériaux de renforcement. De ce point de vue on distingue trois types principaux de composites : les composites renforcés par des particules, les composites stratifiés et les composites renforcés par des fibres.

Les possibilités que présente le contrôle des composites par des techniques ultrasonores non destructives et les problèmes posés par ce type de contrôle ont relancé l'intérêt des études de propagation dans de tels milieux. En effet, à l'intérieur du composite, les réflexions multiples aux frontières des composants sont à l'origine d'une dispersion géométrique qui se traduit par une variation de la vitesse de phase des ondes ultrasonores en fonction de la fréquence. Par exemple la forme d'un train d'ondes ultrasonores qui a traversé un composite est fortement altérée, on constate que sa durée est augmentée et que la fréquence initiale est parfois modifiée; le milieu donne même naissance à un signal « avant-coureur » (ou précurseur) irrégulier dont la période d'oscillation est plus rapide que celle de l'émission.

Dans de telles conditions il devient difficile, voire impossible, de mesurer avec précision la vitesse de phase des ondes ultrasonores. En contrôle non destructif des matériaux il en résulte une incertitude considérable sur la localisation et l'appréciation de l'ordre de grandeur des défauts. C'est pourquoi l'étude expérimentale de la dispersion de vitesse dans un composite stratifié nous a paru intéressante. Afin 
de mieux maîtriser les phénomènes et pour des raisons d'ordre technologique, nous avons réalisé des composites à deux phases résine-aluminium avec des épaisseurs de l'ordre du millimètre.

Ce choix nous a placés dans la gamme des fréquences de 0,1 à 2,5 MHz. Nous avons obtenu une courbe de dispersion de la vitesse de phase, en fonction de la fréquence, qui comprend deux branches correspondant à deux modes de propagation. De nombreuses théories prévoient ces deux modes et nous verrons qu'en fonction des hypothèses choisies, elles ajustent telles ou telles parties de nos résultats.

Dans le prochain paragraphe nous allons décrire le dispositif expérimental de mesure des vitesses et présenter nos résultats.

Dans le paragraphe 3 nous exposerons rapidement trois théories qui dans le cas de composites stratifiés prévoient deux modes de propagation. Pour conclure nous comparerons et discuterons nos résultats avec ceux prévus par ces différentes théories.

\section{Etude expérimentale. - 2.1 RÉALISATION DES} COMPOSITES STRATIFIÉS. - Afin de bien maîtriser leur structure d'une part, et de pouvoir travailler dans la gamme des fréquences ultrasonores comprises entre 0,1 et $2,5 \mathrm{MHz}$ d'autre part, nous avons réalisé nos échantillons de composites stratifiés. Ils sont constitués de plaques d'aluminium d'égale épaisseur réunies entre elles par des couches de résine époxyde de même épaisseur. La photographie de la figure 1 donne deux exemples de structures ainsi réalisées. Elles peuvent simuler des matériaux composites industriels avec une échelle comprise entre 1 et 50 .
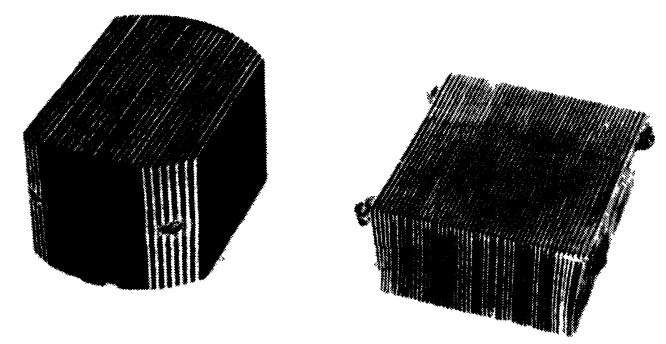

Fig. 1. - Exemples de composites stratifiés.

[Example of laminated composite.]

Les plaques d'aluminium sont découpées en rectangles égaux, leur planéité est contrôlée au marbre et leur surface est soigneusement dégraissée afin d'obtenir une bonne adhérence ultérieure de la résine époxyde. Elles sont percées aux quatre angles puis assemblées par des tiges filetées et des entretroises qui ménagent un intervalle constant entre elles. Cette structure est ensuite placée dans un moule contenant de la résine époxyde liquide (Résine STRM 2 plus durcisseur 954) qui se polymérise en 24 heures. Après démoulage le composite ainsi obtenu est usiné à la fraiseuse afin d'y ménager deux plans parallèles entre eux et perpendiculaires aux plaques d'aluminium. Ces plans constitueront les faces d'entrée et de sortie du signal ultrasonore.

Par la suite nous avons étudié trois structures dont les dimensions sont consignées dans le tableau I :

Tableau I. - Dimensions des composites stratifiés aluminium-résine époxyde.

[Dimensions of the aluminium-epoxy laminated composites.]

\begin{tabular}{|l|c|c|}
\hline & Aluminium & Epoxyde \\
\hline Epaisseurs (mm) & 0,5 & 0,5 \\
Longueurs (mm) & 24,8 & 24,8 \\
\hline Epaisseurs & 0,9 & 0,8 \\
Longueurs & 38 & 38 \\
\hline Epaisseurs & 2 & 2 \\
Longueurs & 50 & 50 \\
\hline
\end{tabular}

2.2 TeChNique DE MESURE. - C'est une technique par transmission [1, 2]. Nous avons calculé la vitesse de propagation des ultrasons à partir d'une mesure de temps de propagation. La gamme de fréquence explorée s'étend de 0,1 à $2,5 \mathrm{MHz}$. Le signal émis toutes les $2 \mathrm{~ms}$ est un train d'ondes d'une durée de $20 \mu \mathrm{s}$. Nous avons utilisé des transducteurs émetteur et récepteur à large bande, placés face à face dans une cuve de mesure remplie d'eau dont la température est contrôlée au $1 / 100^{\mathrm{e}}$ de degré $\mathrm{C}$. Le composite est placé entre les transducteurs, comme le montre la photographie de la figure 2 :

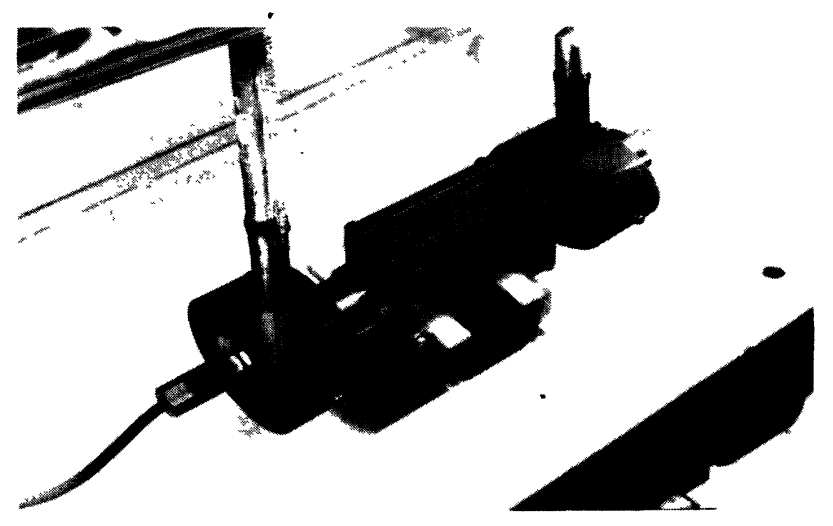

Fig. 2. - Banc pour la mesure des vitesses par transmission. [Bench for velocity measurement by transmission.] 
Il n'est pas au contact des transducteurs mais en est séparé par deux lignes de retard en eau de $1 \mathrm{~cm}$ d'épaisseur. La propagation s'effectue parallèlement aux couches du composite. La photographie de la figure 3 présente une vue générale du banc de mesure :

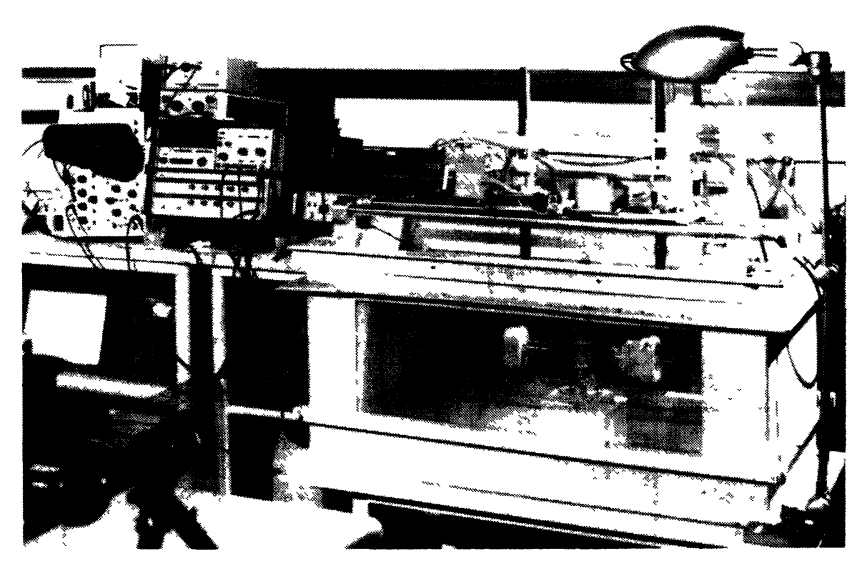

Fig. 3. - Vue générale du dispositif expérimental.

[General view of the experimental set up.]

On y distingue à gauche : l'ensemble des appareils d'émission-réception, un synthétiseur de fréquence, un intervallomètre numérique, deux générateurs d'impulsions, des amplificateurs, et un oscilloscope à 4 voies dont seulement 3 voies sont utilisées. A droite : la cuve de mesure comportant les chariots de positionnement de l'émetteur et du récepteur ultrasonore aìnsi que le plateau support du composite immergé.

Un diviseur de fréquence (rapport $1 / 2000$ ) donne, à partir d'une fréquence fixe de $1 \mathrm{MHz}$ issue du synthétiseur, des signaux de synchronisation qui déclenchent simultanément les deux générateurs d'impulsions et l'oscilloscope. Dans ces conditions nous observons sur l'écran de l'oscilloscope des signaux parfaitement stables :

en A le train d'ondes électriques (signal émis);

en $\mathrm{B}$ le train d'ondes acoustiques (signal reçu);

en $\mathrm{C}$ une impulsion mobile à retard ajustable (précision $10^{-8} \mathrm{~s}$ ). La figure 4 schématise les signaux observés et la figure 5 donne le bloc-diagramme de l'installation de mesure.

Pour mesurer la vitesse de propagation nous avons opéré comme suit : l'impulsion mobile (trace C) est placée successivement sur un "point de phase", préalablement choisi, du train d'ondes reçu (trace B) puis sur le point de même phase du train d'ondes émis (trace A). Le retard est affiché automatiquement sur un intervallomètre associé à un compteur-calculateur qui donne la moyenne de 1000 mesures. Le temps de parcours de l'onde ultrasonore pour traverser le composite s'écrit simplement :

$$
t_{e}=t_{\mathrm{B}}-t_{\mathrm{A}}-t_{\mathrm{R}} \pm t_{0}
$$
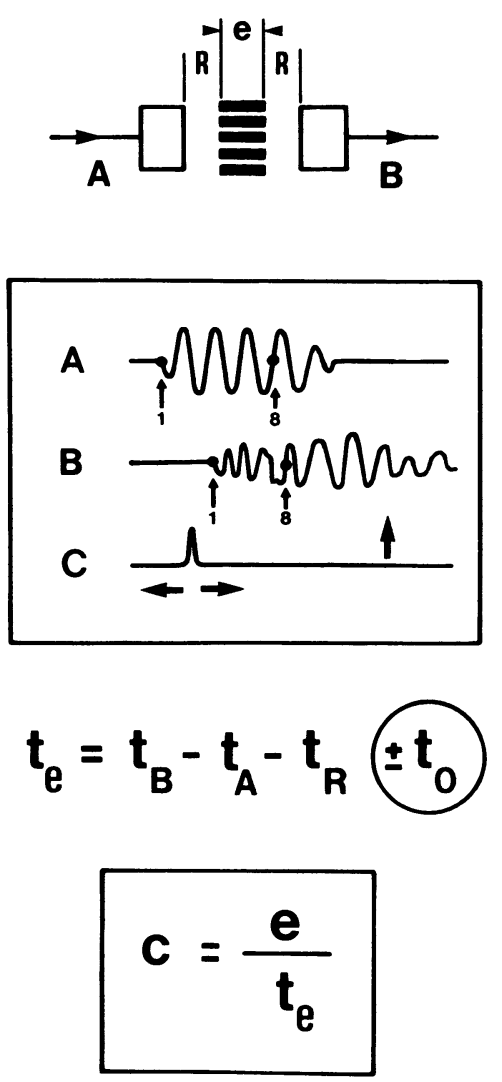

Fig. 4. - Diagramme schématique de la mesure du temps de parcours.

[Schematic diagram for travel times measurement.]

avec

$t_{\mathrm{B}}=$ retard (en $\left.\mu \mathrm{s}\right)$ lu sur la trace $\mathrm{B}$;

$t_{\mathrm{A}}=\operatorname{retard}(\mathrm{en} \mu \mathrm{s})$ lu sur la trace $\mathrm{A}$;

$t_{\mathrm{R}}=$ retard introduit par les 2 lignes de retard en eau;

$t_{0}=$ terme correctif indépendant du composite à étudier et qui, pour chaque fréquence, tient compte des avances ou retards apportés par la chaîne de mesure.

Dans ces conditions la vitesse de phase des ondes ultrasonores dans le composite est donnée simplement par:

$$
c=e / t_{e}
$$

où $e$ est l'épaisseur du composite.

Cette méthode, bien que très simple, permet d'effectuer des mesures correctes malgré les fortes distorsions qui altèrent le signal reçu dans la bande des fréquences qui correspondent à la zone de forte dispersion du composite. Ce point est examiné dans le paragraphe qui suit.

2.2.1 Choix du point de phase. - Lorsque nous avons utilisé cette méthode sur des matériaux homogènes, ne déformant pas le train d'ondes, le choix de ce point n'était pas crucial : en effet, le temps mesuré était le même quel que soit le point choisi, car toute 


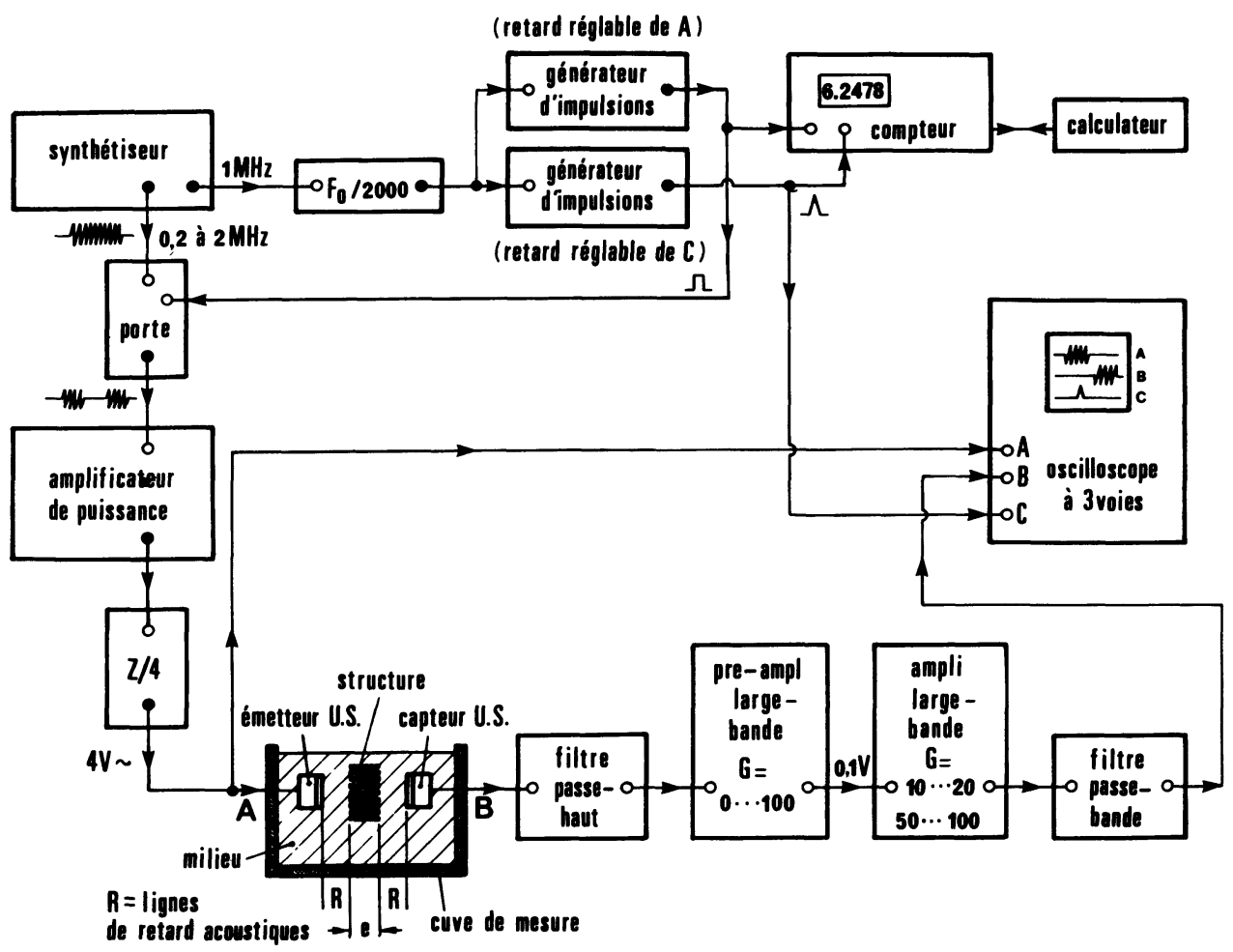

Fig. 5. - Bloc diagramme de l'installation de mesure de vitesse.

[Schematic diagram of experimental arrangement for velocity measurement.]

période ajoutée sur le train d'ondes B était compensée par la même période ajoutée sur le train d'ondes A $\left(t_{\mathrm{B}}-t_{\mathrm{A}}=\right.$ constante $)$.

La précision, comparée aux résultats obtenus par d'autres méthodes, s'est avérée être de l'ordre de $1 \%$.

- Quand nous avons mesuré le temps de transit de l'onde ultrasonore dans des structures à tiges, à billes ou à cavités cylindriques, [1, 2] nous avons constaté, au début du train d'ondes, l'apparition à certaines fréquences - d'une déformation qui ne comportait que deux ou trois oscillations rapides et de faible amplitude (précurseur). Dans ces conditions, pour mesurer la vitesse de phase, il suffisait de choisir un point de phase situé au-delà de la perturbation, par exemple au quatrième zéro.

Pourquoi choisir un zéro ? Parce qu'il est indépendant de l'amplitude de chaque période (souvent irrégulière en B) et que leur emplacement est parfaitement défini par l'intersection de la ligne isoélectrique de l'impulsion de mesure (voie $\mathrm{C}$ ) avec les périodes des trains d'ondes - fussent-elles de faible amplitude et déformées - lorsque l'impulsion est placée successivement sur les voies $B$ et $A$.

- Dans le cas des structures feuilletées (l'onde ultrasonore se déplaçant alors parallèlement aux plans) nous avons constaté, au moment de l'apparition du mode rapide, que la perturbation s'étendait audelà du quatrième zéro. Nous avons alors choisi le huitième zéro pour effectuer des mesures correctes de vitesse.
Choisir un point encore plus éloigné du début du train d'ondes présentait le risque de se placer dans une zone où apparaissent des échos parasites (aller et retour dans la structure par exemple). Cela nous aurait également obligé d'allonger la durée du train d'ondes pour conserver le nombre de périodes nécessaire à la mesure, aux basses fréquences.

2.3 RÉSUltats EXPÉRIMENTAUX. - Nous avons étudié trois structures dont les paramètres géométriques sont rappelés dans le tableau $\mathrm{I}$, et dont les paramètres mécaniques sont donnés dans le tableau II :

Tableau II. - Paramètres acoustiques des deux composants : aluminium-résine époxyde.

[Acoustical parameters of the two constituents aluminium and epoxy.]

\begin{tabular}{|l|l|c|c|}
\hline & & Aluminium & Epoxyde \\
\hline $\mathrm{m} \cdot \mathrm{s}^{-1}$ & $v_{\mathrm{L}}:$ vitesse long. & 6360 & 2770 \\
$\mathrm{~m} \cdot \mathrm{s}^{-1}$ & $v_{\mathrm{t}}:$ vitesse de cis. & 3130 & 1363 \\
$\mathrm{~N} \cdot \mathrm{m}^{-2}$ & $\mu:$ module de cis. & $2,65 \times 10^{10}$ & $0,216 \times 10^{10}$ \\
$\mathrm{~N} \cdot \mathrm{m}^{-2}$ & $\lambda:$ et de Lamé & $6,10 \times 10^{10}$ & $0,458 \times 10^{10}$ \\
$\mathrm{~kg} \cdot \mathrm{m}^{-3}$ & $\rho:$ masse vol. & $2,7 \times 10^{3}$ & $1,16 \times 10^{3}$ \\
\hline
\end{tabular}

Pour ces trois structures nous avons mesuré la vitesse de phase des ondes ultrasonores dans la gamme de fréquences 0,2 à 2,5 MHz. Les croix (reliées par un 
trait fin) placées sur les figures 6,7 et 8 correspondent aux résultats expérimentaux :

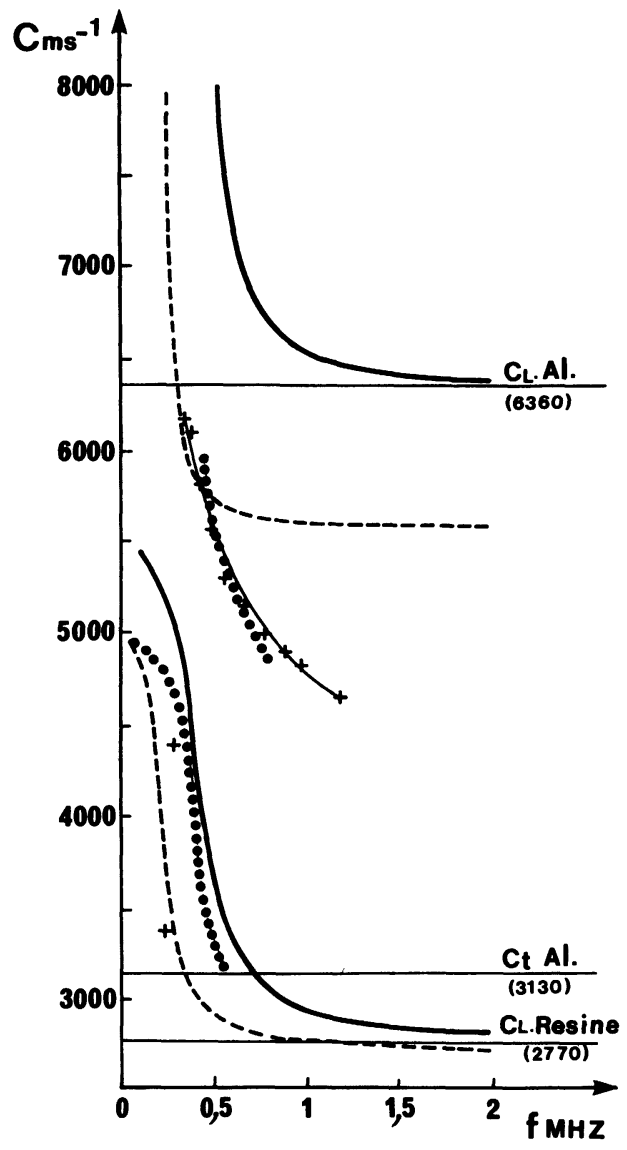

Fig. 6. - Courbes de dispersion pour le stratifié $2+2 \mathrm{~mm}$ (aluminium + résine). $\ldots .=$ Théorie du mélange; $-=$ Théorie simplifiée; $+-+-+-=$ Valeurs expérimentales.

[Dispersion curves of $2+2 \mathrm{~mm}$ aluminium + epoxy laminate. ${ }^{0000}=$ Classical theory $; \cdots=$ Mixture theory; $-=$ Simplified theory $;+-+-+-=$ Experimental data.]

Du côté des basses fréquences nous observons que la vitesse décroît de la valeur $5250 \mathrm{~m} \cdot \mathrm{s}^{-1}$ jusqu'à environ $4500 \mathrm{~m} \cdot \mathrm{s}^{-1}$, elle augmente ensuite brusquement, bien au-dessus des valeurs précédentes, avant de décroître régulièrement pour atteindre une valeur d'environ 4500,5000 ou $5500 \mathrm{~m} \cdot \mathrm{s}^{-1}$ selon l'épaisseur décroissante des plaques utilisées et pour la fréquence la plus élevée qui représente notre dernier point de mesure.

La fréquence correspondant au saut brutal de vitesse dépend de l'épaisseur des plaques d'aluminium et de résine époxyde. Cette fréquence augmente quand les épaisseurs diminuent. Nos résultats mettent en évidence deux modes de propagation ; un mode lent ne présentant pas de fréquence de coupure (qué nous n'avons pas pu mesurer avec assez de précision dans le cas des plaques de $2 \mathrm{~mm}$, Fig. 6) et un mode rapide

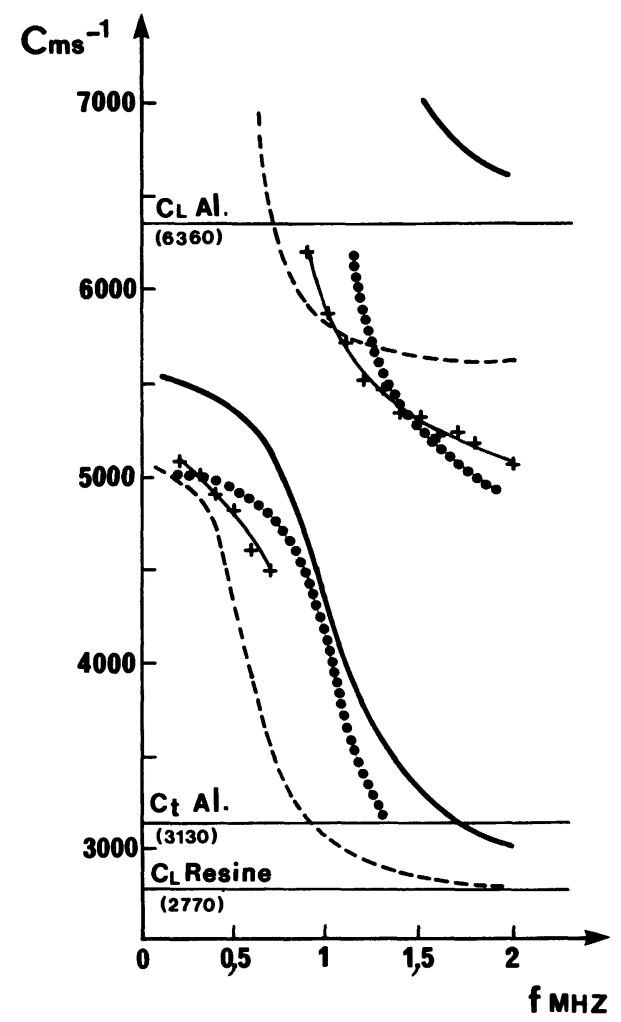

Fig. 7. - Courbes de dispersion pour le stratifié $1+1 \mathrm{~mm}$ (aluminium + résine).

[Dispersion curves of $1+1 \mathrm{~mm}$ aluminium + epoxy laminate.]

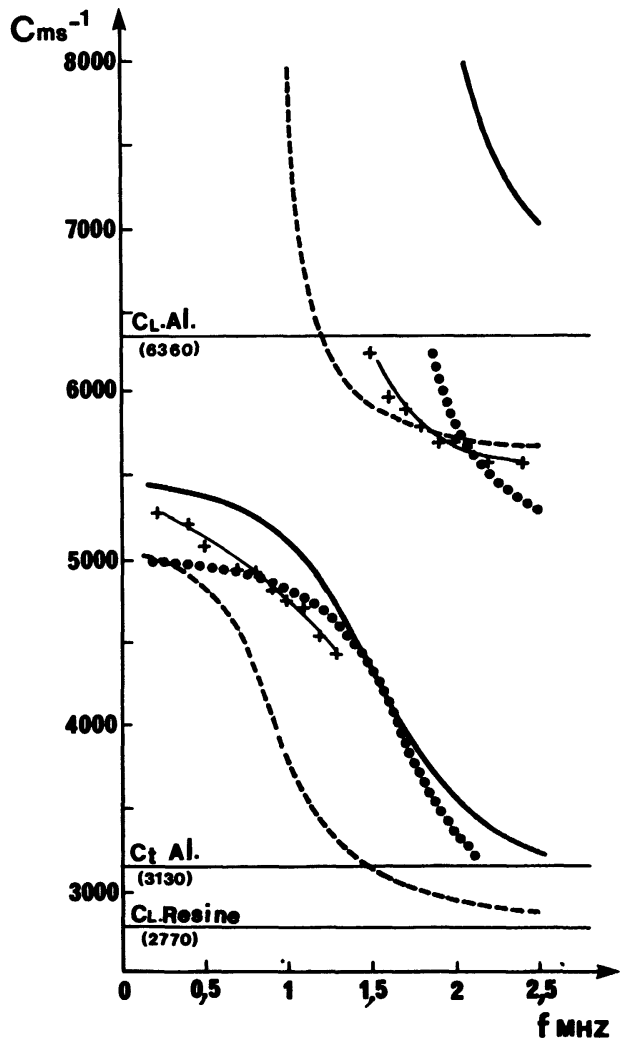

Fig. 8. - Courbes de dispersion pour le stratifié $0,5+0,5 \mathrm{~mm}$ (aluminium + résine)

[Dispersion curves of $0.5+0.5 \mathrm{~mm}$ aluminium + epoxy laminate.] 
possédant une fréquence de coupure. C'est pour cette fréquence que se produit le saut de vitesse que nous. avons déjà signalé. Pour des valeurs supérieures à cette fréquence de coupure les deux modes coexistent mais le retard de propagation entre les deux signaux n'est pas suffisant pour isoler les deux modes. Le signal du mode lent est superposé au signal du mode rapide et l'atténuation étant élevée, il ne nous a pas été possible d'augmenter la longueur du composite afin d'obtenir une bonne séparation des signaux.

Voyons maintenant s'il est possible d'apporter une explication théorique à ces observations.

3. Etude de la dispersion de vitesse. - Notre intention n'est pas de présenter une étude théorique détaillée de la dispersion de vitesse. Au contraire nous avons préféré appliquer directement à nos conditions de mesure le résultat de trois théories choisies parmi les nombreuses qui existent.

- La première due à L. M. Brekhovskikh [3] est dite théorie classique. La solution du problème doit vérifier les conditions aux limites entre couches et conduit à une équation de dispersion assez difficile à manier.

- La deuxième proposée par G. A. Hégemier et al. [4] conduit à une relation explicite donnant la vitesse de propagation en fonction de la fréquence.

- Enfin, dans sa théorie simplifiée A. Ben Chaala aboutit également à une relation explicite entre la vitesse et la fréquence.

Dans la suite nous avons préféré conserver les notations propres à chaque auteur. Dans chaque cas les différents coefficients sont explicités en annexe. Le lecteur intéressé par plus de détails pourra se référer aux publications signalées et retrouvera, avec les mêmes notations, les relations que nous avons mises en ouvre. La figure 9 précise la géométrie et les coordonnées du problème.

Le matériau de renforcement est constitué par l'aluminium, couche indice 1 sur la figure. La matrice est

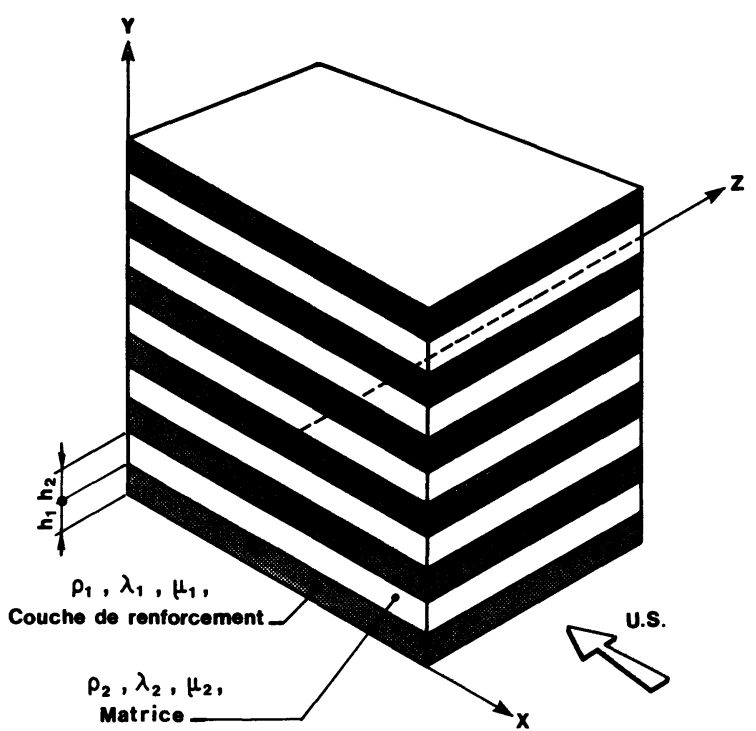

Fig. 9. - Géométrie du composite et système de coordonnées.

[Laminated composite geometry and coordinate system.]

constituée par la résine époxyde, couche indice 2 sur la figure.

3.1 LA THÉORIE CLASSIQUe. - Dans la théorie classique on envisage une onde guidée à l'intérieur des couches qui composent la structure. Cette onde se propage parallèlement aux couches suivant la direction Ox (Fig. 9). On suppose qu'elle est couplée à une onde transversale dont la vibration est parallèle à la direction $\mathrm{O} y$. Suivant cette direction et dans chaque couche, il s'établit un régime d'ondes stationnaires.

La solution du problème doit vérifier les conditions de continuité pour les contraintes et les déformations à la limite de séparation des différentes couches. Dans ces conditions L. M. Brekhovskikh obtient la relation de dispersion suivante :

$$
\begin{aligned}
\omega^{2} \bar{\rho}\left[c^{2} \bar{\rho}+4(\mu-\bar{\mu})\right] X \operatorname{tg} \frac{\bar{\beta} h_{2}}{2}-\omega^{2} c^{2} \rho \bar{\rho}\left[Y \operatorname{tg} \frac{\bar{\beta} h_{2}}{2}+\bar{Y} \operatorname{tg} \frac{\beta h_{1}}{2}\right]= & \\
& -4(\mu-\bar{\mu})^{2} \cdot X \bar{X}-\omega^{2} \rho\left[c^{2} \rho-4(\mu-\bar{\mu})\right] \bar{X} \operatorname{tg} \frac{\beta h_{1}}{2} .
\end{aligned}
$$

Cette équation de dispersion n'est valable que dans l'hypothèse où le mode qui se propage est symétrique, ce qui donne en moyenne une onde longitudinale dans la direction $O x$. Dans ces conditions, elle permet de calculer les variations de la vitesse de propagation suivant la direction $\mathrm{O} x$ en fonction de la fréquence. Dans l'annexe I, nous donnons l'expression et la signification des différents paramètres.

Nous avons appliqué la relation (3) aux trois structures que nous avons étudiées et dont les paramètres géométriques et mécaniques sont donnés par les tableaux I et II.

Il est important de remarquer que pour certaines valeurs de la vitesse, tels ou tels paramètres $\alpha^{2}, \beta^{2}, \bar{\alpha}^{2}$ et $\bar{\beta}^{2}$ peuvent devenir négatifs. En particulier dans la gamme de fréquence que nous avons étudiée, $\alpha^{2}$ est négatif. Nous écrivons alors :

$$
\alpha=i \omega\left(\frac{1}{c^{2}}-\frac{1}{v^{2}}\right)^{1 / 2} .
$$


Seules les expressions de $X$ et $\bar{Y}$. sont modifiées. Elles s'écrivent :

$$
\begin{aligned}
& X=\frac{\omega^{2}}{c^{2}} \operatorname{tg} \frac{\beta h_{1}}{2}-|\alpha| \beta \operatorname{tgh}|\alpha| \frac{h_{1}}{2} \\
& \bar{Y}=\frac{\omega^{2}}{c^{2}} \operatorname{tg} \frac{\bar{\beta} h_{2}}{2}+|\alpha| \bar{\beta} \operatorname{tgh}|\alpha| \frac{h_{1}}{2} .
\end{aligned}
$$

Elles sont valables dans l'intervalle de vitesse :

$$
3130<c<6360 \mathrm{~m} \cdot \mathrm{s}^{-1} \text {. }
$$

Les bornes correspondent aux vitesses de cisaillement et de compression de l'aluminium. Dans cet intervalle nous avons calculé à l'aide de la méthode de Newton-Raphson les solutions de l'équation (3). Nous avons porté les résultats sur les courbes des figures 6 à 8 (courbes à gros pointillés).

La relation de dispersion (3) donne bien deux modes de propagation.

3.2 LA THÉORIE DU MÉLANGe. - Pour obtenir une équation plus simple, G. A. Hégemier et al. [4] ont remplacé les conditions aux limites entre les couches par un terme de couplage proportionnel au déplacement relatif de deux couches voisines. Ils introduisent les déplacements et les contraintes moyennes dans chaque couche.

Ces valeurs moyennes sont calculées en supposant que les déplacements longitudinaux sont symétriques par rapport au centre de chaque couche et que les déplacements verticaux sont antisymétriques par rapport à ce même centre. Ils obtiennent une équation de dispersion du $4 \mathrm{e}$ degré pour la vitesse de propagation dont la solution est donnée par :

$$
1 / c^{2}=\frac{\left[\frac{v c_{0}^{2}}{\omega^{2}}-\left(c_{1}^{2}+c_{2}^{2}\right)\right] \pm \sqrt{\left[\frac{v c_{0}^{2}}{\omega^{2}}-\left(c_{1}^{2}+c_{2}^{2}\right)\right]^{2}-4\left(1-\frac{v}{\omega^{2}}\right)\left(c_{1}^{2} c_{2}^{2}-c_{3}^{4}\right)}}{2\left(c_{3}^{4}-c_{1}^{2} c_{2}^{2}\right)}
$$

Les différents coefficients sont définis à l'annexe II.

L'expression (6) fait apparaître également l'existence d'un mode lent et d'un mode rapide correspondant respectivement aux signes + et - Nous avons porté sur les figures 6 à 8 les valeurs numériques calculées à partir de l'expression (6) (courbes à pointillés longs).

Le mode lent ne possède pas de fréquence de coupure. D'après la relation (6) quand la fréquence tend vers zéro, la vitesse de propagation tend vers la valeur limite $c_{0}$.

Le mode rapide possède une fréquence de coupure obtenue pour $k=0$; dans ces conditions la fréquence de coupure est donnée par :

$$
\omega_{\mathrm{c}}=\sqrt{v} .
$$

Pour $\omega \rightarrow \infty$ les vitesses limites ont pour valeur :

$$
\frac{1}{c_{ \pm}^{2}}=\frac{\left(c_{1}^{2}+c_{2}^{2}\right) \pm \sqrt{\left(c_{1}^{2}-c_{2}^{2}\right)^{2}+4 c_{3}^{4}}}{2\left(c_{1}^{2} c_{2}^{2}-c_{3}^{4}\right)} .
$$

3.3 LA THÉORIE SIMPLIFIÉE. - Dans le but de simplifier encore le problème, l'un de nous [5] a supposé que l'on pouvait négliger les déplacements verticaux à l'intérieur de la structure. Avec cette nouvelle condition et partant des équations obtenues par Hégemier [4] on obtient la relation de dispersion suivante :

$$
\frac{1}{c^{2}}=\frac{1}{2 q c^{2} z^{2}}\left\{\left[q\left(1+z^{2}\right)-\frac{b^{2}}{\omega^{2}}\left(z^{2}+q\right)\right] \pm\left[\frac{b^{4}}{\omega^{4}}\left(z^{2}+q\right)^{2}+2 q \frac{b^{2}}{\omega^{2}}\left(-z^{4}+q z^{2}+z^{2}-q\right)+q^{2}\left(1-z^{2}\right)^{2}\right]^{1 / 2}\right\} \text {. }
$$

Les différents coefficients sont définis à l'annexe III.

Il importe de noter que la relation de dispersion (8) obtenue dans le cadre de la théorie simplifiée est semblable à celle proposée par $\mathrm{A}$. Bedford et $\mathbf{M}$. Stern [6]. Cest avec les notations de ces auteurs que l'on a écrit l'expression (8).
La relation (8) donne également un mode lent et un mode rapide. Pour le mode lent, la vitesse à l'origine pour $\omega \rightarrow 0$ a pour valeur :

$$
c_{0}=c \sqrt{\frac{\left(z^{2}+q\right)}{1+q}} .
$$


Le mode rapide possède une fréquence de coupure donnée par :

$$
\omega_{\mathrm{c}}=b \sqrt{\frac{1+q}{q}} .
$$

Pour $\omega \rightarrow \infty$; la vitesse limite du mode rapide tend vers la vitesse de phase dans le matériau de renforcement (ici l'aluminium), la vitesse limite du mode lent tend vers la vitesse de phase dans la matrice (ici l'époxyde).

Nous avons également tracé sur les figures 6 à 8 les courbes de dispersion correspondant à la théorie simplifée (courbe à traits continus).

4. Discussion et conclusion. - 4.1 Discussion DES RÉSULTATS. - Il n'existe pratiquement pas d'étude expérimentale systématique de la propagation d'ondes ultrasonores se propageant parallèlement aux couches d'un composite. Cela est dû, nous semble-t-il, à la difficulté d'obtenir des mesures précises et répétitives. Cette difficulté, qui provient du caractère dispersif du milieu, est encore augmentée quand on étudie les composites industriels dont les couches ne sont pas toujours d'égale épaisseur, parallèles entre elles, etc... Toutefois en 1969, J. S. Whittier et J. C. Peck [7] ont pu effectuer des mesures sur le mode lent dans la zone basse fréquence.

Plus tard, H. J. Sutherland et R. Lingle [8] ont mis en évidence les deux modes de propagation, dans la zone intermédiaire de fréquences. Enfin du côté hautes fréquences C. H. Yew et P. N. Jogi [9] ont pu repérer les deux signaux correspondant aux modes lent et rapide.

- Du point de vue théorique, les trois modèles traitent uniquement les modes symétriques. Ils font apparaître le mode lent et le mode rapide (voir les Figs. 6, 7, 8). Le mode lent correspond au premier mode symétrique dans le composite. Son allure est très voisine du premier mode symétrique de plaque (ondes de Lamb). Pour les fortes épaisseurs de couches du composite les courbes du mode lent sont relativement voisines. Quand les épaisseurs diminuent, les courbes s'éloignent les unes des autres. Dans tous les cas, pour les fréquences intermédiaires, la théorie classique et la théorie simplifiée donnent des résultats voisins. Au contraire, du côté des basses fréquences, la théorie simplifiée s'écarte de la théorie classique et de la théorie du mélange qui coïncident à l'origine.

Pour le mode rapide, qui correspond au deuxième mode symétrique, l'accord entre les trois théories est moins bon.

L'accord entre la théorie classique et celle du mélange va cependant en s'améliorant quand on diminue l'épaisseur des couches. La théorie simplifiée donne des résultats qui ne concordent plus avec les deux autres théories.

En fait, la théorie simplifiée correspond au cas où il y a un couplage mécanique lâche entre les différentes couches du composite. Dans ces conditions - et cela est bien visible sur la figure 6 - les vitesses hautes fréquences correspondent aux vitesses de phase dans le matériau massique (aluminium ou résine). Les propagations dans l'aluminium et la résine deviennent alors indépendantes, ce qui est contredit par l'expérience.

La mesure de la vitesse des ondes longitudinales en haute fréquence pourrait donc permettre d'apprécier le degré de couplage, donc la cohésion entre les différents matériaux du composite.

- Sur le plan expérimental et pour les trois structures étudiées, nos mesures sont proches des valeurs données par la théorie classique. Pour le mode lent, nos résultats expérimentaux se situent entre la théorie du mélange et la théorie simplifiée.

Pour le mode rapide ils se placent entre la théorie du mélange et la théorie classique.

4.2 ConClusion générale. - Nos résultats expérimentaux confirment l'existence de deux modes de propagation dans les composites stratifiés.

La théorie classique de L. M. Brékhovskikh ajuste assez bien nos mesures pour les trois structures étudiées.

Pour le mode lent cela place la courbe expérimentale entre la théorie du mélange et la théorie simplifiée.

Pour le mode rapide elle se situe entre la théorie du mélange et la théorie classique (et d'autant plus près de cette dernière que les plaques sont plus épaisses). Par contre il n'y a pas de concordance avec la théorie simplifiée.

En résumé la théorie classique qui introduit le moins d'hypothèses simplificatrices ajuste assez bien l'expérience.

La théorie du mélange ne vérifie l'expérience que pour le mode lent et pour les premières fréquences après la fréquence de coupure; elle s'en rapproche cependant pour les plaques les plus minces (Fig. 8).

Enfin, la théorie simplifiée qui suppose un couplage (entre couches) faible, ne vérifie l'expérience que pour le mode lent.

En fait, tout se passe comme si les couches étaient indépendantes, la propagation étant confinée aux couches métalliques. Le mode rapide serait un mode guidé dans la couche d'aluminium seule. Dans les conditions de l'expérience un couplage aluminiumrésine existe qui modifie l'allure de la courbe du mode rapide. 
Annexes. - A-I PARAMÈTRes de la thÉORIE ClASSIQUe.

$$
\begin{array}{ll}
X=\frac{\omega^{2}}{c^{2}} \operatorname{tg} \frac{\beta h_{1}}{2}+\alpha \beta \operatorname{tg} \frac{\alpha h_{1}}{2} \quad & Y=\frac{\omega^{2}}{c^{2}} \operatorname{tg} \frac{\beta h_{1}}{2}-\bar{\alpha} \beta \operatorname{tg} \frac{\bar{\alpha} h_{2}}{2} \\
\bar{X}=\frac{\omega^{2}}{c^{2}} \operatorname{tg} \frac{\bar{\beta} h_{2}}{2}+\overline{\alpha \beta} \operatorname{tg} \frac{\bar{\alpha} h_{2}}{2} & \bar{Y}=\frac{\omega^{2}}{c^{2}} \operatorname{tg} \frac{\bar{\beta} h_{2}}{2}-\alpha \bar{\beta} \operatorname{tg} \frac{\alpha h_{1}}{2} .
\end{array}
$$

Avec

$v=$ vitesse longitudinale,

$$
\begin{array}{ll}
\alpha=\omega\left(\frac{1}{v^{2}}-\frac{1}{c^{2}}\right)^{1 / 2} \cdot \quad \beta=\omega\left(\frac{1}{b^{2}}-\frac{1}{c^{2}}\right)^{1 / 2} \\
\bar{\alpha}=\omega\left(\frac{1}{\bar{v}^{2}}-\frac{1}{c^{2}}\right)^{1 / 2} \quad \bar{\beta}=\omega\left(\frac{1}{\bar{b}^{2}}-\frac{1}{c^{2}}\right)^{1 / 2}
\end{array}
$$

$b=$ vitesse de cisaillement,

$\mu=$ coefficient de Lamé,

$\rho=$ masse volumique,

$h=$ épaisseur des couches.

Les paramètres surlignés correspondent à la matrice (résine époxyde).

A-II Paramètres de la THÉORIE DU MÉLANGE.

$$
\begin{aligned}
& c_{0}^{2}=\left(c_{11}+c_{22}+2 c_{12}\right) / \rho \\
& c_{1}^{2}=c_{11} / \rho_{1}^{(\rho)} ; \quad c_{2}^{2}=c_{22} / \rho_{2}^{(\rho)} ; \quad c_{3}^{4}=c_{12}^{2} / \rho_{1}^{(\rho)} \rho_{2}^{(\rho)} \\
& \gamma=K\left(\rho / \rho_{1}^{(\rho)} \rho_{2}^{(\rho)}\right) ; \quad K=3 \mu_{1} \mu_{2} /\left(\mu_{1} n_{2}+\mu_{2} n_{1}\right)
\end{aligned}
$$

Avec

$$
\begin{aligned}
& \rho=\rho_{1}^{(\rho)}+\rho_{2}^{(\rho)} . \\
& \rho_{\alpha}^{(\rho)}=n_{\alpha} \rho_{\alpha} ; \quad(\alpha=1,2) \\
& n_{\alpha}=h_{\alpha} /\left(h_{1}+h_{2}\right) ; \\
& v=\gamma /\left(h_{1}+h_{2}\right)^{2} . \\
& C_{\alpha \alpha}=\left(n_{\alpha} E_{\alpha}-\frac{\lambda_{\alpha}^{2}}{E}\right) ; \quad C_{\alpha \beta}=\frac{\lambda_{\alpha} \lambda_{\beta}}{E} .
\end{aligned}
$$

Avec

$$
\begin{aligned}
\alpha, \beta & =1,2 ; \quad \alpha \neq \beta \\
E & =\frac{E_{1}}{n_{1}}+\frac{E_{2}}{n_{2}} \\
E_{\alpha} & =\lambda_{\alpha}+2 \mu_{\alpha}
\end{aligned}
$$

$\lambda$ et $\mu$ coefficients de Lamé.

A-III PARAMÈTRES DE LA THÉORIE SIMPLIFIÉE."

$$
\begin{aligned}
c^{2} & =\frac{n_{1}}{\rho_{1}}\left(\lambda_{1}+2 \mu_{1}\right) ; \\
z^{2} & =\frac{n_{2}}{c^{2} \rho_{2}}\left(\lambda_{2}+2 \mu_{2}\right) ; \\
b^{2} & =\frac{1}{c^{2} \rho_{2}} \frac{3 \mu_{1} \mu_{2}}{\left(h_{1} \mu_{1}+h_{2} \mu_{2}\right)\left(h_{1}+h_{2}\right)} ; \\
q & =\frac{\rho_{1}}{\rho_{2}} ; \\
n_{1} & =\frac{h_{1}}{h_{1}+h_{2}} ; \quad n_{2}=\frac{h_{2}}{h_{1}+h_{2}} .
\end{aligned}
$$

L'indice 1 correspond au matériau de renforcement (aluminium).

L'indice 2 correspond à la matrice (résine époxyde). 


\section{Bibliographie}

[1] Miane, M., M'Hirsi, A., Saul, H., Gazanhes, C., Etude expérimentale de la dispersion de vitesse dans une structure périodique. Acustica 43 (1979) 217-221.

[2] item (deuxième partie). Acustica 47 (1981) 325-332.

[3] Brekhovskikh, L. M., Waves in layered media (Academic Press, New York) 1960.

[4] Hégemier, G. A., Gurtman, G. A., Nayfeh, A. H., A continuum mixture theory of wave propagation in laminated and fiber reinforced composites. Int. J. Solids Struct. 9 (1973) 395-414.

[5] Ben ChaAla, A., Propagation d'ondes ultrasonores dans des milieux composites types renforcés de fibres et stratifiés. Communication au Séminaire National sur le contrôle non destructif, Alger, 14-16 avril 1980.
[6] Bedford, A., Stern, M., Toward a diffusing continuum theory of composite materials. J. Appl. Mech. 38 (1971) 8-14.

[7] WhittieR, J. S. and PECK, J. C., Experiments on dispersive pulse propagation in laminated composites and comparison with theory. J. Appl. Mech. 36 (1969) 485-490.

[8] Sutherland, H. J. and Lingle, L., Geometric dispersion of acoustic waves by a fibrous composite. J. Compos. Mater. 6 (1972) 490-502.

[9] YEW, C. H. and JoGI, P. N., A study of wave motion in fiber-reinforced medium. Int. J. Solids Struct. 12 (1976) 693-703. 